\title{
Identification of ABSCISIC ACID (ABA) signaling related genes in Panax ginseng
}

\author{
Jeongeui Hong $\cdot$ Hogyum Kim $\cdot$ Hojin Ryu
}

Received: 10 December 2018 / Revised: 10 December 2018 / Accepted: 10 December 2018

(C) Korean Society for Plant Biotechnology

\begin{abstract}
Korean ginseng (Panax ginseng) has long been cultivated as an important economic medicinal plant. Owing to the seasonal and long-term agricultural cultivation methods of Korean ginseng, they are always vulnerable to various environmental stress conditions. ABSCISIC ACID (ABA) is an essential plant hormone associated with seed development and diverse abiotic stress responses including drought, cold and salinity stress. By modulating ABA responses, plants can regulate their immune responses and growth patterns to increase their ability to tolerate stress. With recent advances in genome sequencing technology, we first reported the functional features of genes related to canonical ABA signaling pathway in $P$. ginseng genome. Based on the protein sequences and functional genomic analysis of Arabidopsis thaliana, the ABA related genes were successfully identified. Our functional genomic characterizations clearly showed that the ABA signaling related genes consisting the ABA receptor proteins $(P g P Y L s)$, kinase family ( $P g S n R K s)$ and transcription factors ( $P g A B F s, P g A B I 3 s$ and $P g A B I 5 s)$ were evolutionary conserved in the $P$. ginseng genome. We confirmed that overexpressing ABA related genes of $P$. ginseng completely restored the $\mathrm{ABA}$ responses and stress tolerance in $\mathrm{ABA}$ defective Arabidopsis mutants. Finally, tissue and age specific spatio-temporal expression patterns of the identified ABArelated genes in $P$. ginseng tissues were also classified using various available RNA sequencing data. This study provides ABA signal transduction related genes and their functional genomic information related to the growth and development of Korean ginseng. Additionally, the results of this study could be useful in the breeding or artificial selection of ginseng which is resistant to various stresses.
\end{abstract}

J. Hong $\cdot$ H. Kim $\cdot$ H. Ryu $(\bowtie)$

Department of Biology, Chungbuk National University, Cheongju 28644, Republic of Korea

e-mail: hjryu96@chungbuk.ac.kr
Keywords Panax ginseng, ABA, Drought stress, Signal transduction, Gene expression

\section{Introduction}

$P$. ginseng C. A. Meyer is an important perennial herb plant belonging to Araliaceae. It is a worldwide medicinal plant used for over thousand years in Asia, especially in Korea, Japan and China (Hu 1976). Many studies and clinical trials have demonstrated the efficacy of ginseng, including cancer prevention, HIV virus replication inhibition, and aging inhibition (Shin et al. 2000, Choi et al. 2008). The cultivation of ginseng is a very slow growth rate and requires 4 to 6 years of cultivation period to obtain sufficient active ingredients (Chung et al. 2016). It is susceptible to environmental stresses such as growth environment, salt in soil, pests, drought and high temperature because it has to grow for a long time (Liu et al. 2014). In addition, the yield of ginseng has been greatly reduced due to recent global warming with cold weather, drought, and high temperature. Since the frequency of occurrence of an abnormal climate is increasing, the necessity of varieties cultivating highly adaptable environment is required.

Plants are sessile living organisms that have evolved a variety of signaling mechanisms to respond to and cope with ever-changing environments. Representative reactions are through plant hormones such as brassinosteroid, jasmonic acid, ethylene, abscisic acid (ABA) (Kim et al. 2016, Verma et al. 2016). Plants respond to a variety of stresses through these plant hormones, and also tolerate to environmental stresses such as cold weather or dryness through ABA signaling pathways (Nakashima and Yamaguchi-Shinozaki 2013, Saddhe et al. 2017). The ABA response is mediated by SnRK, which acts as a kinase, with transcription factors including ABAINSENSITIVE 3, 4 and 5 to modulate the expression of downstream target genes (Nakashima and Yamaguchi-Shinozaki 2013). This response is not inhibited by negative regulators, 
ABA-INSENSITIVE 1 and 2 (ABI1, 2), which is type 2c protein phosphatases (PP2C), unless stress conditions are present. However, when plants are under stress condition, the biosynthesis of ABA is rapidly increased and the canonical ABA signaling is activated by the receptors, PYRABACTIN RESISTANCE 1(PYR1)/PYR1-LIKE(PYL)/REGULATORYCOMPONENTS OF ABA RECEPTORS (RCAR) (Nakashima and YamaguchiShinozaki 2013). The combined PYR1 / PYL / RCAR and ABA complexes directly bind with ABA-INSENSITIVE 1, 2 $(A B I 1,2)$. When the phosphatase function of ABI1 and ABI2 is inhibited, the $A B A$ reaction occurs because the inhibition of SNF1-RELATED PROTEIN KINANS (SnRKs) is released (Umezawa et al. 2009). In many studies, these ABA signaling related gene has been used to generate stress resistant plants (Sah et al. 2016). Therefore, molecular breeding using ginseng's ABA signal transduction gene will be able to cultivate environmentally resistant varieties. However, much research has been done on the genome of ginseng, but ginseng is a allotetraploid plant $(2 n=4 x=48)$ and has a larger genome size (3.2 Gbp) (Hong et al. 2004, Choi et al. 2014, Jang et al. 2017). So the research on ginseng was focused on pharmacological research and tissue culture (Yang and Yang 2000, $\mathrm{Kim}$ et al. 2009). Recent advances in genome sequencing technology have led to the detoxification of genomes and transcripts in $P$. ginseng, which could be used for functional genomic approaches (Jiang et al. 2017, Jo et al. 2017, Waminal et al. 2018).

In this study, ABA signaling related genes in $P$. ginseng were identified from recently reported draft genome and transcript data (Jiang et al. 2017, Jo et al. 2017, Waminal et al. 2018). The expression patterns of the identified ABA signaling related genes in $P$. ginseng were examined in the different age and tissues. We also found that the expression was regulated by exogenous $\mathrm{ABA}$, suggesting that they are involved in $\mathrm{ABA}$ signal transduction. It was also confirmed that the $\mathrm{ABA}$ responsiveness was restored by complementation of the ginseng ABA related genes in ABA signaling defective Arabidopsis mutants.

\section{Materials and Methods}

Phylogenetic tree construction, classification expression profiles analysis of ABA related genes from $P$. ginseng

The protein sequences of ABA-related genes were selected from previous studies (Jiang et al. 2017; Jo et al. 2017; Waminal et al. 2018). A phylogenetic tree based on protein sequence alignment was generated using MEGA version 7.0 software by the neighbor-joining method with a bootstrap value of 1000 (Hall et al. 2013, Kumar et al. 2016). An online program, iTOL (http://itol.embl.de/), was applied to beautify the phylogenetic tree. Base on the phylogenetic tree constructed by ABA related genes from $P$. ginseng and Arabidopsis, these genes were divided into different groups and subgroups. The expression values were calculated by $\log 2$ (FPKM) and were presented in heat map using XLSTAT software.

ABA treatments and Real Time qRT-PCR

One-year-old ginseng roots were incubated with water containing $100 \mu \mathrm{M}$ ABA (Sigma) for 3, $6 \mathrm{~h}$. After treatment, the samples were immediately frozen in liquid nitrogen. Total RNAs were extracted from seedlings using a Total RNA extraction kit (Taesin Bioscience, Korea) according to the manufacturer's instructions. Total RNA concentration and quality were measured using a K5600 Micro-spectrophotometer (Shanghai Biotechnol Co., China). A first-strand synthesis kit (Enzynomics, Korea) with oligo (dT) primers was used for cDNA synthesis from $1 \mathrm{ng}$ of total RNA. The cDNA was then used for real-time quantitative PCR with a Quant Studio 3 (Applied Biosystems, USA) instrument using SYBR Green Real-time PCR Master Mix (Applied Biosystems). Primer lists are followed : PgTUB1 : 5'-GGCGAAATCTTCGAGA ATGC-3' and 5'-TCGAAACCCTAACAAAAGAAAAGG-3'; PgABIla : 5'-GGTATGTGATATGGCTCGAAAGC-3' and 5'-TCCTTGCTGCCCTTTTGAAG-3'; PgABI2a: 5'-CCAA TGAGGAGGTATGTGACACA-3' and 5'-TTCCCTTTTGA AGAGCTCGATT-3'; PgABF1 : 5'-ACGAGCTCGGAAAC AGGCTTA-3' and 5'-CTTTTCCAAGGCATTTTCATCTTC-3'; $P g P Y L 1$ : 5'-GTCATTGAAGGAAGACCTGGTACTC-3' and 5'-TGCAAGCCGCTCTGAAACTT-3'; PgPYL2 : 5'-GAGC ATATTCTTAGCGTCAGGATTG-3' and 5'-GGTGTTCCC TTCTGGCACAT-3'; PgPYL3 : 5'-TCATGGATGGGAGAC CAGGTA-3' and 5'-CAGCCAAACGCTCAGAGACA-3'; PgPYL4: 5'-AGTCATTACTGTCCATCCCGAAGT-3' and 5'-GCCA GTGATTTGAGGTTGCA-3'; PgPYL5 : 5'-TCAGTCATTA CTGTCCATCCTGAAG-3' and 5'-CAGTGATTTGAGGTT GCACTTGA-3'; PgSnRK1 : 5'-GCGAATCTGATAAACCC ACACA-3' and 5'-CACTCTCTAGGTCGTCCTCCATGT-3'; PgSnRK2 : 5'-TGATCAGCAGCCAGTTTGAAGA-3' and 5'TCTCAGGATCGGAGTCCAAGTC-3'; PgSnRK3 : 5'-GAG CAACCCATGCAGAGCAT-3' and 5'-CCTCTCCACTGCT GTCAATATCAA-3'; Threshold cycle $(\mathrm{Ct})$ values were used to calculate $2^{-\Delta \Delta \mathrm{Ct}}$ for expression analysis, where $\Delta \Delta \mathrm{Ct}$ for treated plants was determined as follows: (Ct target gene - $\mathrm{Ct}$ actin gene) - control plant (Ct target gene - $\mathrm{Ct}$ actin gene) (Livak and Schmittgen 2001). 
Protoplast transient expression assay

The full-length cDNAs of PgPYLA, PgPYL5, PgSnRK1, PgSnRK2, $P g S n R K 3, P g A B F 1, A R R 2$ were cloned into plant expression vectors containing $H A, F L A G$ or GFP DNA sequence tags in the $C$ terminus driven by the $35 S: C 4 P P D K$ promoter as previously described (Ryu et al. 2007). For protoplasts transient expression assays, about $4 \times 10^{4}$ protoplasts were transfected with $20 \mu \mathrm{g}$ of plasmid DNA and then incubated under constant light condition at $20^{\circ} \mathrm{C}$ for $6 \mathrm{~h}$. For the subcellular localization, GFP-tagged constructs were transfected into protoplasts. ARR2-RFP was used as a nuclear marker. GFP and RFP fluorescence were observed with a fluorescence microscope (Nikon).

Plant materials and growth conditions

Arabidopsis thaliana ecotype Col-0 was used as wild-type controls, and rcar, snrk2.2/2.3 and snrk2.6 knock out mutants were used as the genetic backgrounds of transgenic lines. Arabidopsis seeds were germinated on solid media ( $\mathrm{pH}$ 5.75.8) containing 1/2 Gamborg B5 salts (Duchefa, Netherlands), $1 \%$ sucrose and $0.8 \%$ plant-agar and all plants were grown in a greenhouse under long-day conditions (16-h light/8-h dark cycles) at $22^{\circ} \mathrm{C}$.

Transgenic plants and immunoblotting assay

To generate transgenic plants overexpressing HA-tagged PgPYL3, 4 and PgSnRK1, 3 in the rcar, snrk2.2/2.3, snrk2.6 mutant background, respectively, the gene fragment was cloned into $p C B 302 E S$ or $p C A M B I A 1303$ containing the $35 \mathrm{~S}$ promoter and double HA tag sequences as described previously (Ryu et al., 2014). Electroporation was used to introduce the PgPYL3-pCAMBIA1303, PgPYLA-pCAMBIA1303 and PgSnRK1pCB302ES, PgSnRK3-pCB302ES vector into Agrobacterium strain GV3101. All transgenes were integrated into the Arabidopsis genome by Agrobacterium-mediated floral dipping methods. Transgene expression was verified by immunoblotting. Total proteins from 5-days-old seedlings were extracted with protein extraction buffer (50 mM Tris- $\mathrm{HCl}$ (pH 7.5), $75 \mathrm{mM}$ $\mathrm{NaCl}, 5 \mathrm{mMEDTA}, 1 \mathrm{mM}$ dithiothreitol, $1 \times$ protease inhibitor cocktail (Roche), and 1\% Triton X-100). Total protein was subjected to SDS-PAGE (10\% polyacrylamide), transferred to a PVDF membrane and immunodetected with $1 / 2,000$ dilution of peroxidase-conjugated high-affinity anti-HA (Roche) antibodies.
Physiological analysis of germination and drought stress responses

For seed germination assays, surface-sterilized homozygous seeds were kept at $4^{\circ} \mathrm{C}$ for 3 days and plated on $1 / 2$ Gamborg B5 medium containing $1 \%$ sucrose supplemented with or without the ABA. The seed germination (radicle emergence and green cotyledon) rates were scored. For leaf water loss assays, leaves were detached from 16 days after germination Arabidopsis plants and placed under the greenhouse conditions. Leaf fresh weight was measured immediately after separation, then leaves were placed in Petri dishes and weight was measured over time.

\section{Results and Discussion}

Identification of ABA signaling related components in $P$. ginseng genome

To identify the genes involved in ABA signal transduction in the $P$. ginseng genome, we analyzed two recently reported full-length draft genome sequence information (Choi et al. 2014, Jiang et al. 2017 Waminal et al. 2018) and transcript data using SMRT PacBio sequencing technique (Jo et al. 2017). The genes predicted to play a role for ABA signaling in ginseng by blast method using the protein sequence information of genes involved in ABA signaling confirmed by functional genomic studies in Arabidopsis and several model systems. We identified total putative 67 protein sequences which could be ABA signaling components including 29 PYR1 / PYL / RCARs as ABA receptor proteins (PgPYL1 to PgPYL29), the negative regulatory 7 phosphatase (PP2C) $\mathrm{ABI} 1$ and 2 of ABA signaling (PgABI1a to PgABI1c, PgABI2a to PgABI2d), 14 phosphorylated enzyme SnRKs (PgSnRK1 $\sim$ PgSnRK14), 5 transcription factor $\mathrm{ABI} 3$ (PgABI3a to PgABI3e), 4 ABI4 (PgABI4a to PgABI4d), 12 ABFs (PgABF1 to PgABF12) and $6 \mathrm{ABI} 5$ (PgABI5a to PgABI5f), respectively (Table 1). The gene sequence information revealed that much number of ABA signal transduction related genes are presented in the $P$. ginseng genome compared to that of other plants. It is possible that this feature of $P$. ginseng is an evolutionary product to maintain the characteristics of heterozygous dielectrics and the ability of ginseng to cope with various external stresses with whole lifetime of more than 100 years. For the further functional genomic study of these predicted genes, the evolutionary similarity among the genes was confirmed based on the protein sequence information of ABA signal transduction genes of Arabidopsis (Fig. 1). As shown in Figure 1, the protein 
Table 1 Identified ABA-related genes in the genome of Panax ginseng

\begin{tabular}{|c|c|c|}
\hline Gene name & [TAIR]Description & Total \\
\hline ABI1 & ABA INSENSITIVE 1 & \multirow{2}{*}{7} \\
\hline $\mathrm{ABI} 2$ & ABA INSENSITIVE 2 & \\
\hline $\mathrm{ABI} 3$ & ABA INSENSITIVE 3 & 5 \\
\hline $\mathrm{ABI} 4$ & ABA INSENSITIVE 4 & 4 \\
\hline SnRKs & SNF1-RELATED PROTEIN KINASE family & 14 \\
\hline PYR/PYL/RCAR & REGULATORY COMPONENT OF ABA RECEPTOR family & 29 \\
\hline ABFs & ABSCISIC ACID RESPONSIVE ELEMENT-BINDING FACTOR family & 12 \\
\hline ABI5 & ABA INSENSITIVE 5 & 6 \\
\hline
\end{tabular}

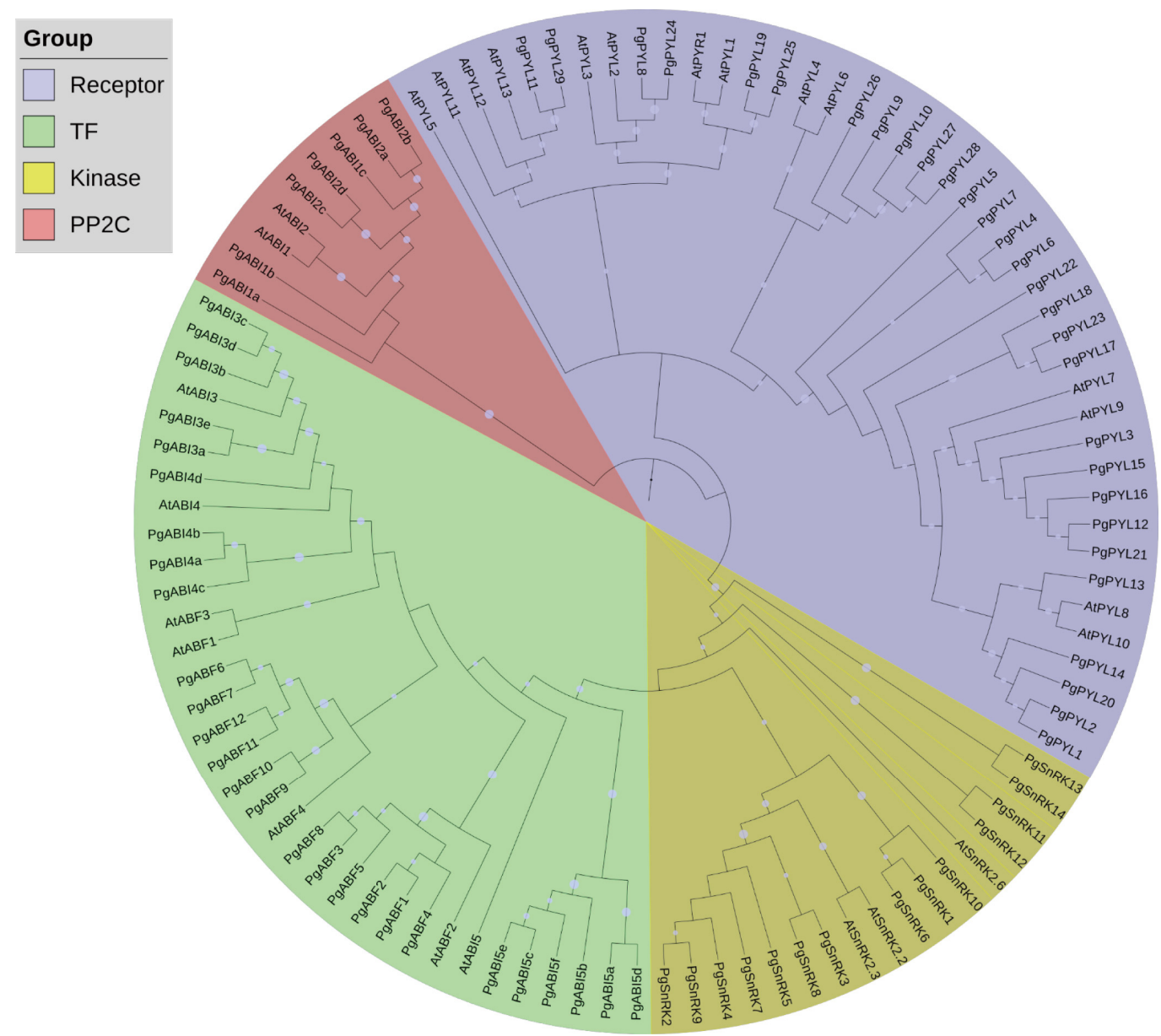

Fig. 1 Phylogenetic tree analysis of ABA related genes from $P$. ginseng and $A$. thaliana genome. The Neighbor-joining tree was constructed with ABA related genes from $P$. ginseng and A. thaliana using MEGA7.0 with a bootstrap of 1000

structure of genes identified from ginseng genomic information is related to those of the ABA signaling system in Arabidopsis model plants, which are consisted with the functional receptors, transcription factors (TF), and phosphorylation related functions (Kinase, PP2C). These results strongly support the possibility that the ABA signaling system to cope with diverse external environmental stresses is well preserved evolutionarily in the
P. ginseng genome.

Spatio-temporal expression patterns of ABA signaling related genes

We next confirmed that the $\mathrm{ABA}$ signaling related genes were expressed in the different ginseng tissues by reanalyzing 
A

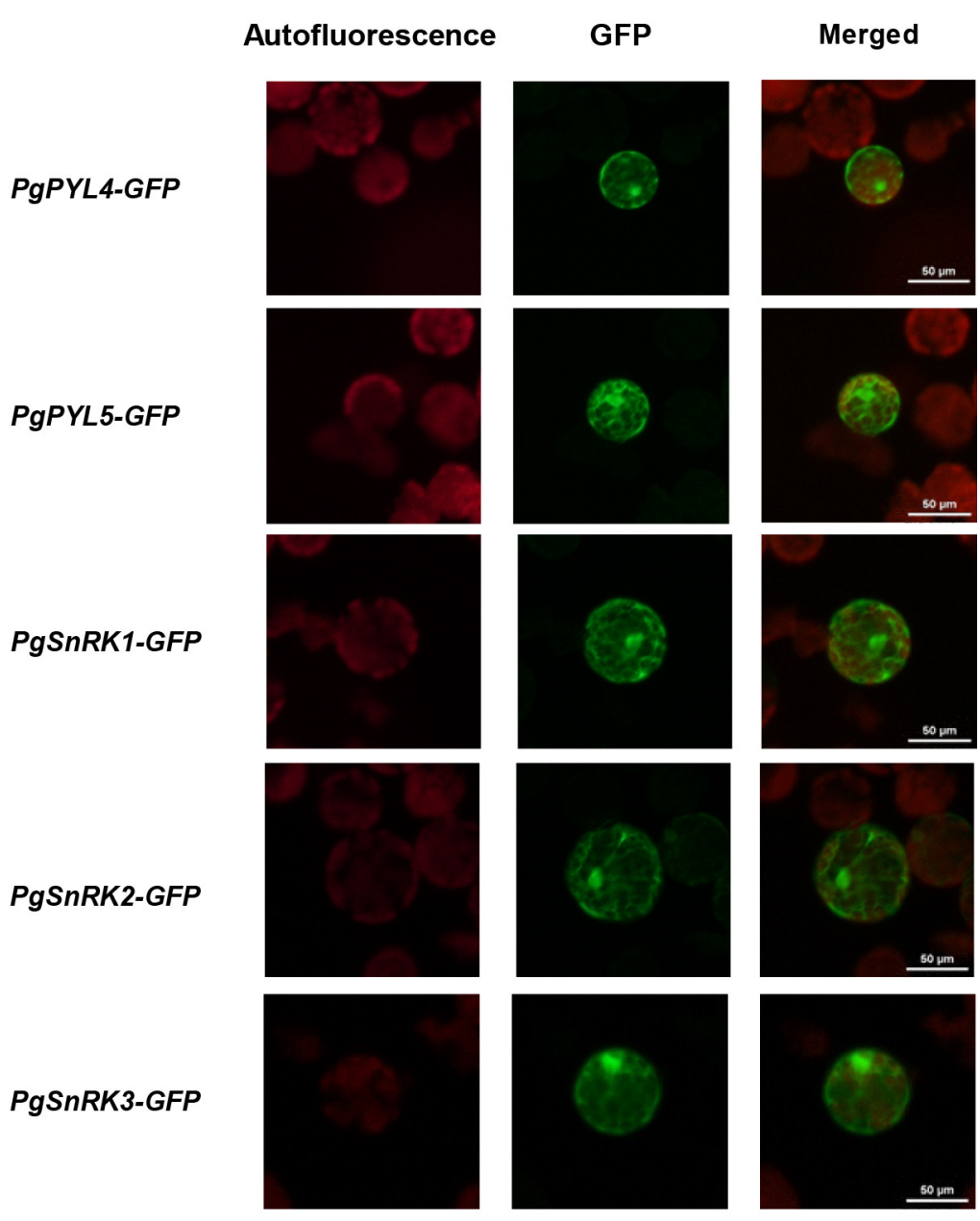

B

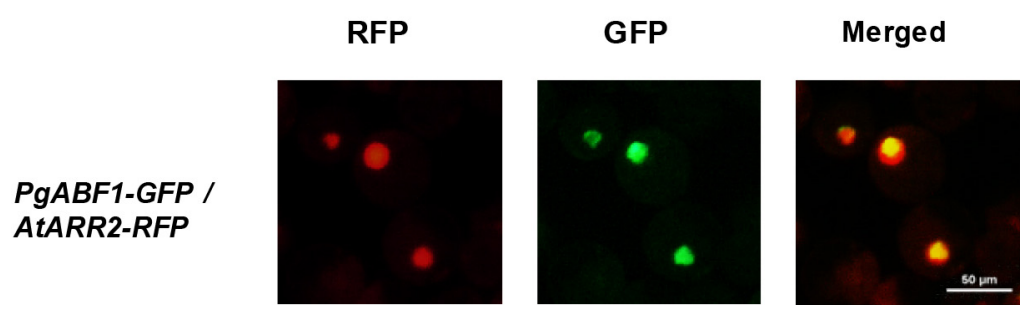

Fig. 2 Heat map of spatio-temporal expression patterns of ABA related genes in different tissues of $P$. ginseng. Heat map of the expression levels of ABA-related genes from $P$. ginseng in 2, 3, 4, 5 and 6 years old roots (A) and different tissues (B). (C) The relative expression levels of PgABF1-4, PgABIIa, PgABI2a, PgPYL1-5 and PgSnRK1-3 in P. ginseng root treated with ABA 100 $\mu \mathrm{M}$ for 0,3 , and 6 hours. Green, high expression; red, low expression; black, intermediate

available RNA-seq data in NCBI (Liu et al. 2017) and our unpublished data (Fig. 2). Relative levels of gene expression were determined by ginseng growth period $(2,3,4,5$ and 6 year old roots, Fig. 2A) and different ginseng tissues including root developmental stages, stem, leaf tissues, fruits and seeds (Fig. 2B). In case of PgSnRKs, most genes were highly expressed during early stage of root developments, but PgSnRK13 and 14 were gradually increased in the late 4-6 years old roots (Fig. 2A). ABA receptors (PgPYLs) and transcription factors $(P g A B F$ s and $A B I 3 \mathrm{~s}$ ) were specifically expressed during early and late root developmental stages. It was also found that the genes expressed in the upper part and the lower part of ginseng plants were differently regulated in the ginseng tissues. For example, the expressions of PgSnRKs were ubiquitously detected in most tissues, but $P g A B I s$ were mostly expressed in the root tissues (Fig. 2B). However, $P g A B I 1$ and $P g A B I 2$ were rarely expressed in 2 and 3 year olds, but not in late stage of rood developments. $P g A B I 3 s$ showed lower expression patterns in 2-year-old ginseng roots. Interestingly, the expression patterns of $P g A B I 5 s$ in the early stages of development were observed, 
but it was decreased in the late stages of development (Fig. 2A). These age and tissue specific expression patterns were similarly observed in the other $\mathrm{ABA}$ signaling related genes. These results confirmed that the expression of $\mathrm{ABA}$ signaling related genes are correlated with almost coincided with that of seed germination and stress responses. To confirm the ABA responsive gene expression, the expression level of each gene in ginseng treated with $\mathrm{ABA}$ for $0,3,6$ hours was analyzed by real-time quantitative PCR (qRT-PCR) (Fig. 2C). $A B F s$ are well known as an ABA responsive gene, and $P g A B F s$ were upregulated by exogenous ABA. PYL / PYR/RCAR showed a decrease in expression when the activity of ABA was increased (Fujii et al. 2009, Ma et al. 2009). Similary to this previous report, the expression of $\mathrm{ABA}$ receptors was down-regulated by ABA. The expression pattern of PP2Cs (PgABIIa and 2a) and SnRK was also decreased by ABA treatments (Fig. 2C). These results suggest that the ABA signal transduction genes are tissue specifically expressed in ginseng and their expression is regulated by ABA, suggesting that these genes are indeed involved in ABA signal transduction pathways in $P$. ginseng.

Subcellular localization of $P$. ginseng ABA signaling components

To confirm the protein localization in the cells, where they are similar to the protein localization of ABA signaling genes of Arabidopsis, we next determined the subcellular localization of GFP tagged genes in the protoplasts (Fig. 3). It has been well characterized that PYLs and SnRKs are localized in both cytoplasm and the nucleus in plant cells (Fujita et al. 2009, Ma et al. 2009). We successfully transfected the PgPYL4-GFP, PgPYL5-GFP, PgSnRK1-GFP, PgSnRK2-GFP and PgSnRK3GFP into Arabidopsis protoplasts (Fig. 3A). When GFP expression was confirmed, all PYLs and SnRKs of $P$. ginseng were clearly detected in the nuclear and cytoplasm similar to Arabidopsis. The transcription factor, ABFs in Arabidopsis have been reported to be expressed in the nucleus since they are transcription factors (Yoshida et al. 2010, Yoshida et al. 2015). In order to confirm that PgABF1-GFP of ginseng is actually expressed in the nucleus, it was confirmed by expression with AtARR2-RFP (Lohrmann et al. 2001) (Fig. 3B). It was confirmed that ARR2-RFP and PgABF1-GFP were co-localized in the nucleus according to the coincidence of intracellular expression positions. These results suggest that ABA signal transduction genes identified in ginseng may play the same roles as previously reported in Arabidopsis research.

Functional analysis of $P$. ginseng ABA signaling genes

To determine the functional roles of the identified genes in
ABA signal transduction, the germination rate and resistance to drought stress were investigated with $\mathrm{Col}-0$ and overexpressing lines in the rcar, snrk2.2/2.3 and snrk2.6 Arabidopsis genetic mutant backgrounds in the presence of ABA (Fig. 4). We initially confirmed the transgene expression level by a western blotting and germination rates of all wild type control and transgenic lines in the absence of ABA (Fig. 4A, 4C and 4E). All tested seeds were completely germinated within 4 days after sowing (DAS), indicating the rare inhibition effects of ABA related genes in the transgenic plants. However, the germination rate of Col- 0 was almost $20 \%$ or less, but that of rcar and snrk $2.2 / 2.3$ mutants was over $90 \%$ at 5DAS in the presence of ABA (Fig. 4B, 4D). Nonetheless, the complementation lines which were overexpressed with PgPYL3, 4 (rcar 35S:PgPYL3-HA and rcar 35S:PgPYL4-HA) and PgSnRK3 (snrk2.2/2.3 35S:PgSnRK3-HA \# 4 and 6) were completely restored the $\mathrm{ABA}$ responses as much as a wild type Col-0 control (Fig. 4B, 4D). These results suggest that PgPYL3, 4 and PgSnRK3 are involved in ABA signal transduction. Surprisingly, the germination rate was not recovered by only overexpression of PgSnRK1-HA in the snrk2.2/2.3 mutant background in the medium containing ABA (Fig. 4D). The PgSnRK1 is similar to AtSnRK2.6 (Fig. 1), being a protein known to play a major role in drought stress due to involvement in stomata aperture rather than seed germination controls (Yoshida et al. 2002, Fujii et al. 2007). To test this possibility, we next evaluated if the drought stress susceptibility of the snrk2.6 (ost1) plants was recovered by complementation of AtSnRK2.6 function by overexpression of PgSnRK1. The resistance to the drought stress was determined by measuring water loss rate of leaf with the snrk2.6 35S: PgSnRK1-HA \# 1 and 5 plants (Fig. 4E). The water loss of detached leaves from 16 old plants was weighed for 2 hours. After 2 hours, the water loss of the Col- 0 plant was reduced by $44 \%$ compared with that of the immediately after cutting, but the snrk 2.6 plants decreased by $58 \%$. However, the water loss of two independent snrk2.6 35S: PgSnRK1-HA transgenic plants significantly was recovered by $49 \%$ and $50 \%$ at 2 hours, respectively (Fig. 4E). Taken together, ABA signal transduction genes identified from $P$. ginseng in this study have functional similarity with the ABA related genes in Arabidopsis. Recent advances in genome sequencing technology have enabled functional genomic analysis of many different plant species (Choi et al. 2014, Jiang et al. 2017, Jo et al. 2017, Waminal et al. 2018). In this study, we analyzed genes involved in the canonical ABA signal transduction pathway in $P$. ginseng, which play essential roles in broad range of environmental stress tolerances. Much research has been conducted on multi environmental stress tolerant plants 
A

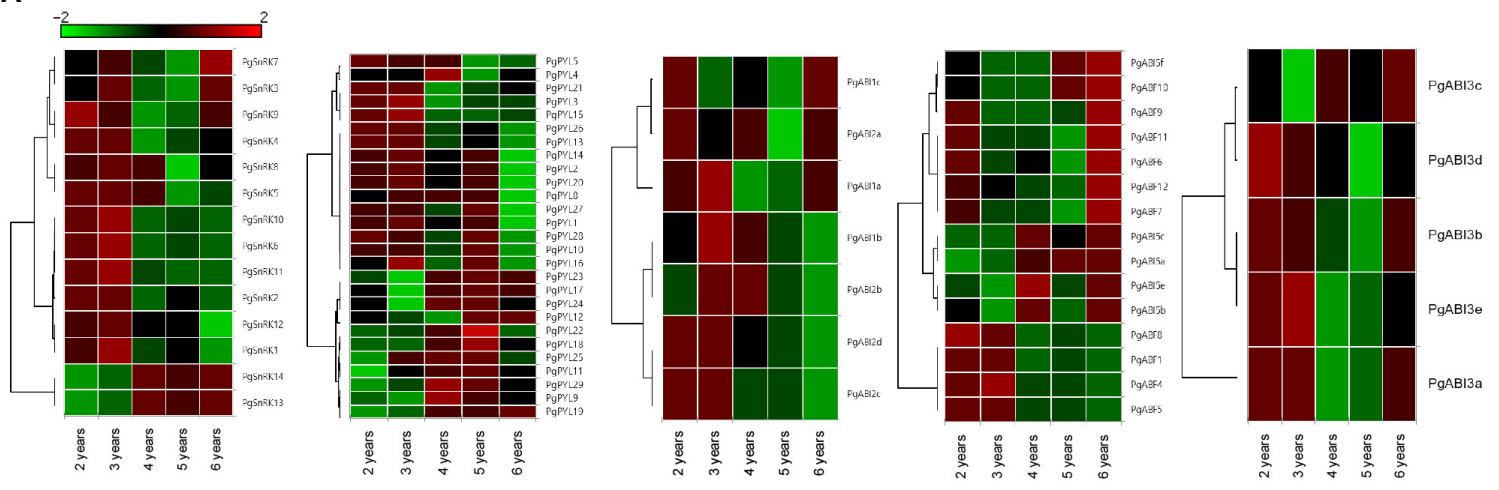

B
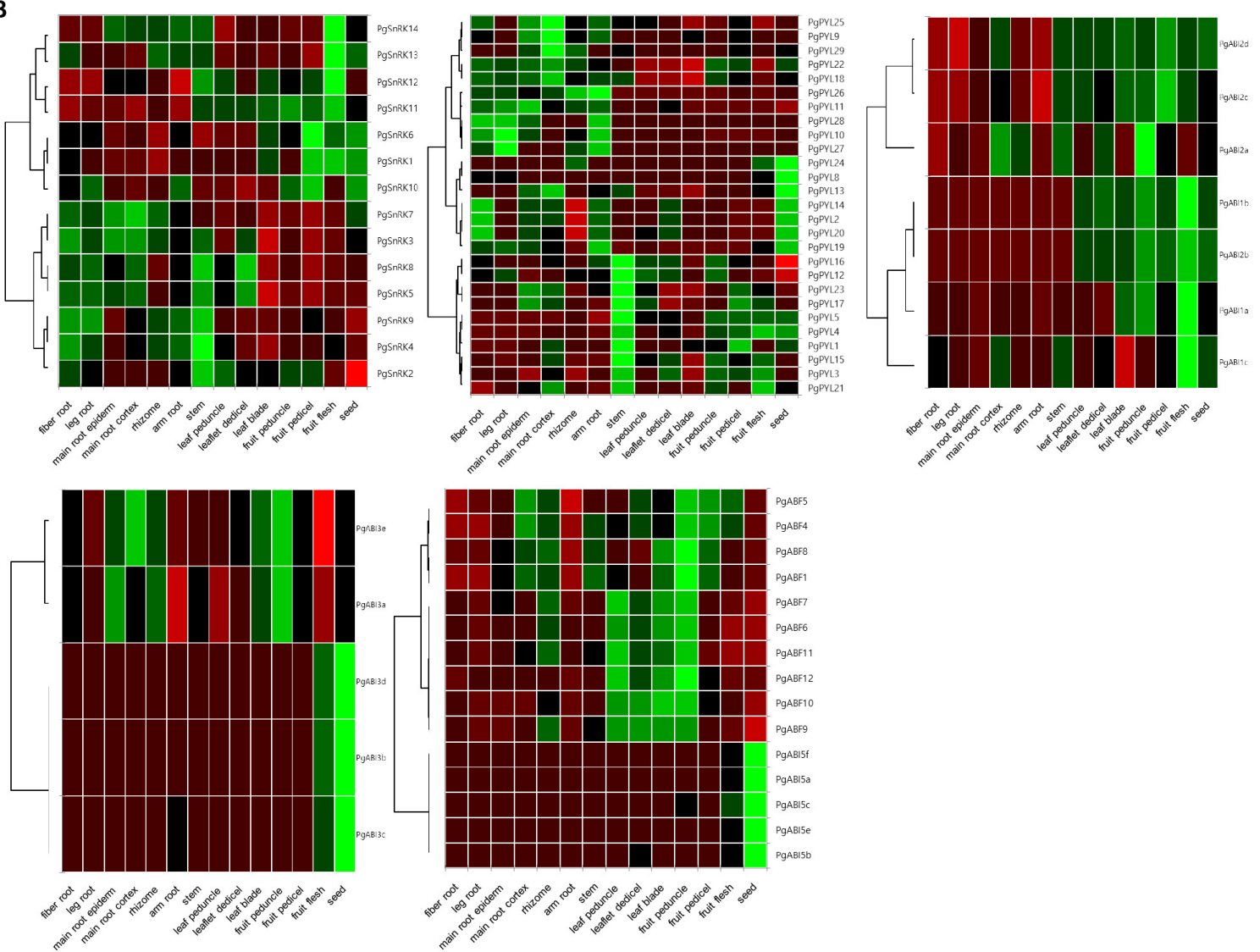

C

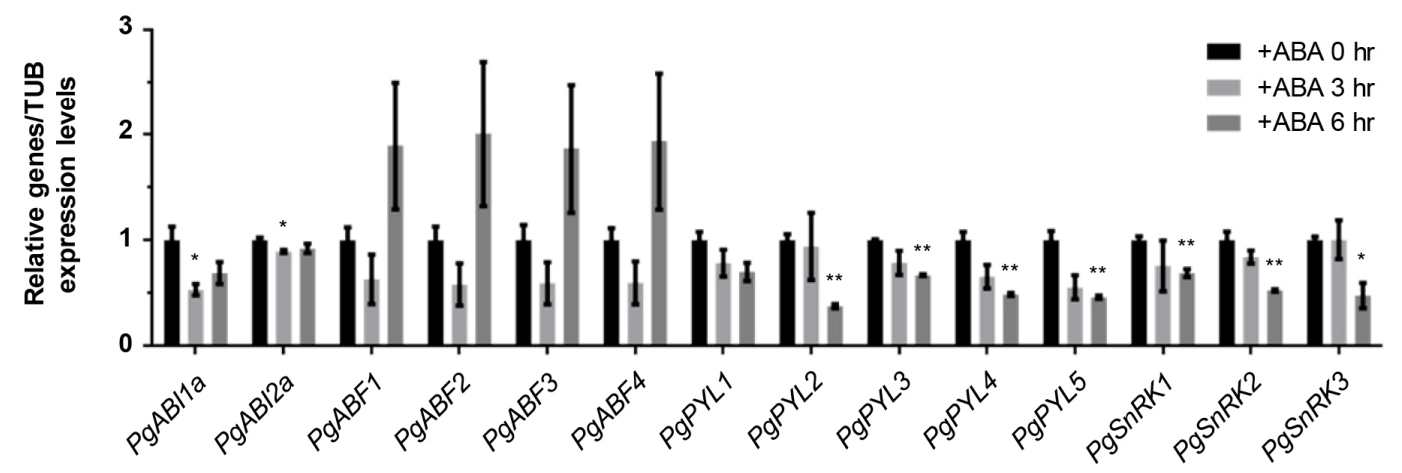

Fig. 3 Subcellular localization of $P$. ginseng ABA related genes in plant cells. Subcellular localization of $P g P Y L 4,5, P g S n R K 1-3$ (A) and $P g A B F 1$ (B) were observed in Arabidopsis mesophyll protoplasts. GFP tagged ABA related genes from $P$. ginseng were transfected into the protoplasts to confirm the subcellular localization. ARR2-RFP was used as a nuclear marker. Scale bar $=50 \mu \mathrm{m}$ 
A

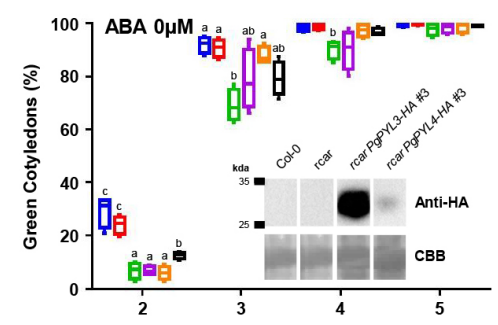

C
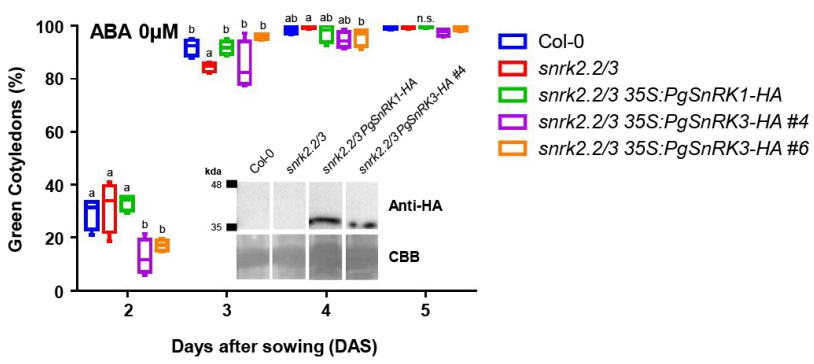

E

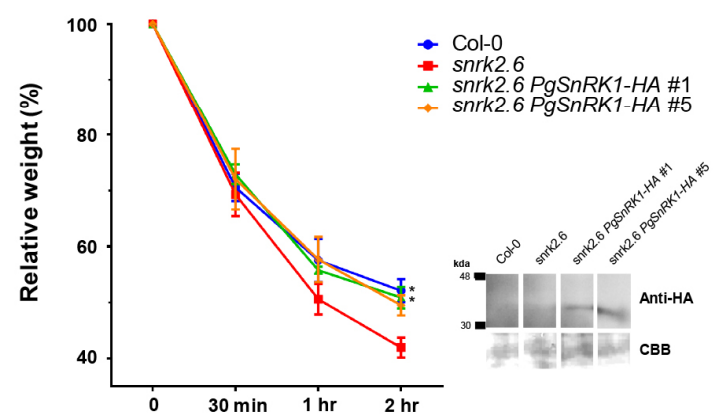

B

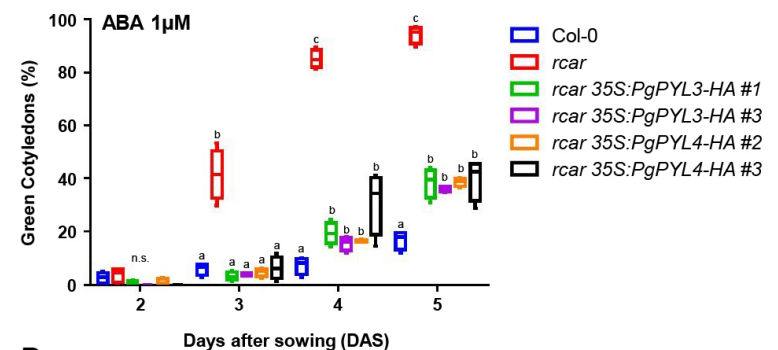

D

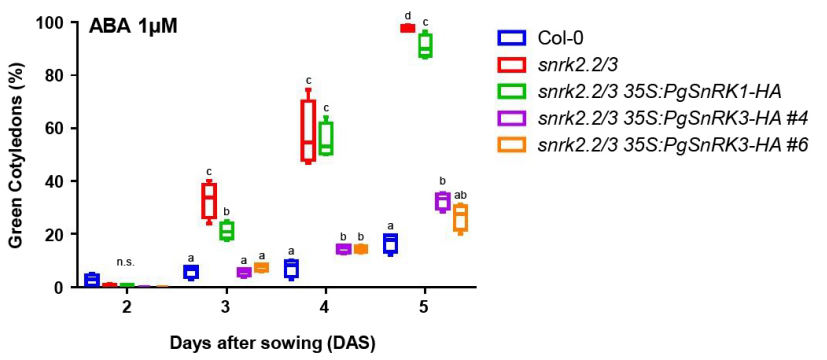

Fig. 4 Overexpression of the $P$. ginseng ABA signaling related genes was completely complemented in the ABA insensitive phenotypes of ABA defective Arabidopsis mutants. The ABA-mediated inhibition of seed germination was measured using green cotyledon emergence. Col-0, rcar, rcar PgPYL and snrk2.2/3 PgSnRK transgenic seeds were germinated on 1/2 B5 agar plates containing $0(\mathrm{~A}, \mathrm{C})$ or $1 \mu \mathrm{M}$ of ABA (B, D). Western blot showing HA-tagged PgPYL3, 4 (A), PgSnRK1, 3 (C), PgSnRK1 (E) proteins from independent transgenic lines. Col-0, negative control; CBB staining indicates loading control. Error bars indicate S.E.M. $(\mathrm{P}<0.05$; one-way ANOVA). (E) Relative water loss rates were measured by weight of leaves from 16 days-old Col-0, ost 1 (snrk 2.6) and two ost1 PgSnRK1 transgenic lines grown under LD $(\mathrm{n}=4)$. Error bars indicate S.E.M ( ${ }^{*} \mathrm{P}<0.05$, Student's t-test)

through molecular breeding due to the extreme climate changes caused by global warming. It is expected that the identified ABA signaling related genes of $P$. ginseng will be able to use in breed ginseng program for increasing resistant to environmental stress.

\section{Acknowledgments}

This work was carried out with the support of the NextGeneration BioGreen 21 Program (No. PJ01313601), Rural Development Administration, Republic of Korea.

\section{Reference}

Choi HI, Waminal NE, Park HM, Kim NH, Choi BS, Park M, Choi D, Lim YP, Kwon SJ, Park BS (2014) Major repeat components covering one-third of the ginseng ( $\mathrm{P}$ anax ginseng $\mathrm{CA}$ Meyer) genome and evidence for allotetraploidy. The Plant Journal 77:906-916

Choi Kt (2008) Botanical characteristics, pharmacological effects and medicinal components of Korean Panax ginseng CA Meyer. Acta Pharmacologica Sinica 29:1109-1118

Chung I-M, Lim J-J, Ahn M-S, Jeong H-N, An T-J, Kim S-H (2016) Comparative phenolic compound profiles and antioxidative activity of the fruit, leaves, and roots of Korean ginseng (Panax ginseng Meyer) according to cultivation years. Journal of ginseng research 40:68-75

Fujii H, Chinnusamy V, Rodrigues A, Rubio S, Antoni R, Park S-Y, Cutler SR, Sheen J, Rodriguez PL, Zhu J-K (2009) In 
vitro reconstitution of an abscisic acid signalling pathway. Nature 462:660

Fujii H, Verslues PE, Zhu J-K (2007) Identification of two protein kinases required for abscisic acid regulation of seed germination, root growth, and gene expression in Arabidopsis. The Plant Cell 19:485-494

Fujita Y, Nakashima K, Yoshida T, Katagiri T, Kidokoro S, Kanamori N, Umezawa T, Fujita M, Maruyama K, Ishiyama K (2009) Three SnRK2 protein kinases are the main positive regulators of abscisic acid signaling in response to water stress in Arabidopsis. Plant and Cell Physiology 50:2123-2132

Hall BG (2013) Building phylogenetic trees from molecular data with MEGA. Molecular biology and evolution 30:1229-1235

Hong C, Lee S, Park J, Plaha P, Park Y, Lee Y, Choi J, Kim K, Lee J, Lee J (2004) Construction of a BAC library of Korean ginseng and initial analysis of BAC-end sequences. Molecular Genetics and Genomics 271:709-716

Hu SY (1976) The genus Panax (ginseng) in Chinese medicine. Economic Botany 30:11-28

Jang W, Kim N-H, Lee J, Waminal NE, Lee S-C, Jayakodi M, Choi H-I, Park JY, Lee J-E, Yang T-J (2017) A glimpse of Panax ginseng genome structure revealed from ten BAC clone sequences obtained by SMRT sequencing platform. Plant Breeding and Biotechnology 5:25-35

Jiang X, Yang C, Baosheng L, Shuiming X, Qinggang Y, Rui B, He S, Linlin D, Xiwen L, Jun Q (2017) Panax ginseng genome examination for ginsenoside biosynthesis. GigaScience

Jo I-H, Lee J, Hong CE, Lee DJ, Bae W, Park S-G, Ahn YJ, Kim Y. C, Kim JU, Lee JW (2017) Isoform sequencing provides a more comprehensive view of the panax ginseng transcriptome. Genes 8:228

Kim H, Moon S, Lee J, Bae W, Won K, Kim Y-K, Kang KK, Ryu $H$ (2016) Identification of multiple key genes involved in pathogen defense and multi-stress tolerance using microarray and network analysis. Journal of Plant Biotechnology 43: 347-358

Kim T-D, Kim Y-S, Han J-Y, Lim S, Choi Y-E (2009) Metabolic engineering for production of ginsenosides in Panax ginseng. Journal of Plant Biotechnology 36:352-359

Kumar S, Stecher G, Tamura K (2016) MEGA7: molecular evolutionary genetics analysis version 7.0 for bigger datasets. Molecular biology and evolution 33:1870-1874

Liu M, Ding W, Gao Y, Li Y (2014) Identification of bacterial strain ge15 and its controlling effect on ginseng diseases. Zhongguo Zhong yao za zhi= Zhongguo zhongyao zazhi= China journal of Chinese materia medica 39:4754-4758

Liu S, Liu M, Wang S, Lin Y, Zhang H, Wang Q, Zhao Y (2017). Analysis of the Panax ginseng stem/leaf transcriptome and gene expression during the leaf expansion period. Molecular medicine reports 16:6396-6404

Livak KJ, Schmittgen TD (2001) Analysis of relative gene expression data using real-time quantitative PCR and the $2-$ $\Delta \Delta \mathrm{CT}$ method. methods 25:402-408

Lohrmann J, Sweere U, Zabaleta E, Baurle I, Keitel C, Kozma-Bognar L, Brennicke A, Schafer E, Kudla J, Harter K
(2001) The response regulator ARR2: a pollen-specific transcription factor involved in the expression of nuclear genes for components of mitochondrial complex $\mathrm{I}$ in Arabidopsis. Molecular Genetics and Genomics 265:2-13

Ma Y, Szostkiewicz I, Korte A, Moes D, Yang Y, Christmann A, Grill E (2009) Regulators of PP2C phosphatase activity function as abscisic acid sensors. Science 324:1064-1068

Nakashima K, Yamaguchi-Shinozaki K (2013) ABA signaling in stress-response and seed development. Plant cell reports 32: 959-970

Ryu H, Kim K, Cho H, Park J, Choe S, Hwang I (2007) Nucleocytoplasmic shuttling of BZR1 mediated by phosphorylation is essential in Arabidopsis brassinosteroid signaling. The Plant Cell 19:2749-2762

Saddhe AA, Kundan K, Padmanabh D (2017) Mechanism of ABA Signaling in Response to Abiotic Stress in Plants. Mechanism of Plant Hormone Signaling under Stress 1:173-195

Sah SK, Reddy KR, Li J (2016) Abscisic acid and abiotic stress tolerance in crop plants. Frontiers in plant science 7:571

Shin HR, Kim JY, Yun TK, Morgan G, Vainio H (2000) The cancer-preventive potential of Panax ginseng: a review of human and experimental evidence. Cancer Causes \& Control 11:565-576

Umezawa T, Sugiyama N, Mizoguchi M, Hayashi S, Myouga F, Yamaguchi-Shinozaki K, Ishihama Y, Hirayama T, Shinozaki K(2009) Type 2C protein phosphatases directly regulate abscisic acid-activated protein kinases in Arabidopsis. Proceedings of the National Academy of sciences 106:17588-17593

Verma V, Ravindran P, Kumar PP (2016) Plant hormone-mediated regulation of stress responses. BMC plant biology 16:86

Waminal NE, Pellerin RJ, Jang W, Kim HH, Yang T-J (2018) Characterization of chromosome-specific microsatellite repeats and telomere repeats based on low coverage whole genome sequence reads in Panax ginseng. Plant Breeding and Biotechnology 6:74-81

Yang DC, Yang KJ (2000) Patterns and Contents of Ginsenoside in Normal Root Parts and Hairy Root Lines of Panax ginseng C. A. Meyer. Journal of Plant Biotechnology 27:485-489

Yoshida R, Hobo T, Ichimura K, Mizoguchi T, Takahashi F, Aronso J, Ecker JR, Shinozaki K (2002) ABA-activated SnRK2 protein kinase is required for dehydration stress signaling in Arabidopsis. Plant and Cell Physiology 43: 1473-1483

Yoshida T, Fujita Y, Maruyama K, Mogami J, Todaka D, Shinozaki K, YAMAGUCHI-SHINOZAKI K (2015) Four A rabidopsis $\mathrm{AREB} / \mathrm{ABF}$ transcription factors function predominantly in gene expression downstream of SnRK2 kinases in abscisic acid signalling in response to osmotic stress. Plant, cell \& environment 38:35-49

Yoshida T, Fujita Y, Sayama H, Kidokoro S, Maruyama K, Mizoi J, Shinozaki K, Yamaguchi-Shinozaki K (2010) AREB1, AREB2, and ABF3 are master transcription factors that cooperatively regulate ABRE-dependent $\mathrm{ABA}$ signaling involved in drought stress tolerance and require $\mathrm{ABA}$ for full activation. The Plant Journal 61:672-685 